\title{
Improving Mobile Video Quality Through Predictive Channel Quality Based Buffering
}

\author{
Jan Willem Kleinrouweler \\ Centrum Wiskunde \& Informatica \\ Amsterdam, The Netherlands \\ j.w.m.kleinrouweler@cwi.nl \\ Hans van den Berg \\ TNO, University of Twente, \\ Centrum Wiskunde \& Informatica \\ The Hague, The Netherlands \\ j.l.vandenberg@tno.nl
}

\author{
Britta Meixner \\ Tiledmedia, Centrum Wiskunde \& Informatica \\ Amsterdam, The Netherlands \\ britta.meixner@cwi.nl
}

Rob van der Mei

Centrum Wiskunde \& Informatica,

$V U$ University Amsterdam

Amsterdam, The Netherlands

r.d.van.der.mei@cwi.nl

\author{
Joost Bosman \\ Centrum Wiskunde \& Informatica \\ Amsterdam, The Netherlands \\ j.w.bosman@cwi.nl
}

\author{
Pablo Cesar \\ Centrum Wiskunde \& Informatica, \\ Delft University of Technology \\ Amsterdam, The Netherlands \\ p.s.cesar@cwi.nl
}

\begin{abstract}
Frequent variations in throughput make mobile networks a challenging environment for video streaming. Current video players deal with those variations by matching video quality to network throughput. However, this adaptation strategy results in frequent changes of video resolution and bitrate, which negatively impacts the users' streaming experience. Alternatively, keeping the video quality constant would improve the experience, but puts additional demand on the network. Downloading high quality content when channel quality is low requires additional resources, because data transfer efficiency is linked to channel quality. In this paper, we present a predictive Channel Quality based Buffering Strategy (CQBS) that lets the video buffer grow when channel quality is good, and relies on this buffer when channel quality decreases. Our strategy is the outcome of a Markov Decision Process. The underlying Markov chain is conditioned on 377 real-world LTE channel quality traces that we have collected using an Android mobile application. With our strategy, mobile network providers can deliver constant quality video streams, using less network resources.
\end{abstract}

Index Terms-Video streaming; HTTP Adaptive Streaming; 5G Mobile Networks; Markov Decision Process; Buffering strategy

\section{INTRODUCTION}

Online video streaming is one of the most popular applications on fixed and mobile networks. Network traffic predictions, such as Sandvine's Internet phenomena report [1], not only show an increase in data volume, but also show that on-demand video streaming is the dominant driver for this increase. Currently, around $70 \%$ of downlink traffic accounts for video streaming. However, mobile networks are a challenging environment to deliver high-quality video. A shared radio-based medium combined with user movement creates high variability in channel quality from eNodeB to User Equipment (UE). The effective throughput to UE is strongly depending on cell load and radio channel conditions.

Currently, Dynamic Adaptive Streaming over HTTP (DASH) is the primary video streaming technique for online video [2]. It has shown to effectively deal with throughput variations [3], [4]. DASH players adapt the video quality to match network throughput. On the one hand, lowering the video quality when network conditions decrease reduces annoying playback interruptions. On the other hand, increasing the video quality when throughput increases provides a benefit for the user. However, frequently streaming at low quality and too many quality switches negatively impact the viewers' Quality of Experience (QoE) [5]. It could even lead to users stopping the stream [6].

The next generation of mobile networks potentially offers better support for video streaming. In 5G networks, so-called network slices can be used to optimize the network for video stream delivery. Having the $5 \mathrm{G}$ architecture in mind, we investigate the potential of a network slice that requests network resources for maintaining a constant video quality. Instead of letting video players adapt their quality to the mobile network conditions, we propose a strategy that adjusts the number of network resources to let players stream at constant video quality.

Streaming at a constant video quality is possible but requires more network resources. In LTE terms this means an increasing usage of Resource Blocks (RBs). The effective data rate per RB strongly depends on the quality of the radio channel between eNodeB to UE. When the channel quality decreases, the eNodeB uses modulation settings with more redundancy, thus effectively reducing the throughput per RB to the UE. Maintaining a constant video quality means using a relatively large number of RBs when channel quality is low.

Traditional adaptation algorithms are efficient, because they lower the video quality when the channel quality decreases, thus relieving the network when it's needed. In this paper, we propose a downloading strategy that also relieves the network when channel quality is low, but it maintains constant video quality. The intuition behind our predictive Channel Quality based Buffering Strategy (CQBS) is to grow the video buffer when channel quality is high (bandwidth is thus relatively cheap), and consume this buffer when channel quality decreases (relieve the network when 
bandwidth becomes expensive). To determine when the buffer should grow or shrink, we compose a Markov chain that represents the changes and behavior of LTE channel quality. The Markov chain is conditioned on an extensive set of 377 minute LTE channel quality traces containing measurements in different environments and under different speeds. We define a Markov Decision Process (MDP) based on this model and the current play-out buffer level in the video player to obtain the optimal downloading strategy. With CQBS we can deliver constant video quality for the same price as the varying video quality from traditional adaptation algorithms. We summarize the contribution in this paper as follows:

- A Markov chain that describes LTE channel quality behavior. The model is conditioned on an extensive set of LTE channel quality traces that we have collected in the real word using an Android application.

- CQBS: a predictive downloading strategy for video streaming (grow-, maintain-, and shrink the video buffer) that reduces network resources while streaming in a constant video quality. We formulate the strategy as an MDP.

- Performance evaluation of CQBS, comparing it to traditional adaptation algorithms. We cross-validate CQBS using our real-world channel quality traces.

The remainder of this paper is structured as follows. Section II discusses related work. In Section III we describe our Markov model for LTE channel quality, which we use in Section IV to compute the optimal downloading strategy. In Section V we evaluate the performance of CQBS. Section VI discusses our findings and concludes this paper.

\section{RELATED WORK}

Dynamic Adaptive Streaming over HTTP (DASH) is the dominant video streaming technology for the Internet [7]. In DASH, a video is encoded at multiple bitrates and resolutions. Each representation is then split into small segments of a few seconds. A manifest file describes the characteristics of each representation and contains URLs where to find each segment [2]. A DASH player downloads the video segments one-byone adapting the video quality to the network conditions. This process is challenging [8], especially in mobile networks that show large throughput variations. The resulting fluctuations in video quality are distracting for the viewers [5] and may even lead to users stopping the stream [6].

To improve the stability of video streams, so called DASH Aware Network Elements (DANEs) are introduced [9]. DANEs are a cross-utilization solution where DASH players exchange information with network elements, such as routers, Wi-Fi access points, and potentially mobile base stations. Most DANEs (or comparable solutions) are targeting wired or WiFi networks. Bouten et al. use proxy servers to guide DASH players [10]. Cofano et al. [11] and Kleinrouweler et al. [12] both presented implementations that employ WebSockets for information exchange. They apply traffic shaping to guarantee that enough bandwidth is available for video streaming.
DANEs also prove to be useful in mobile networks, where they primarily focus on reducing video freezes. In [13], Wirth et al. present a cross-layer solution where the LTE eNodeB has access to the DASH manifest. It allows the eNodeB to better schedule resources and reduce the number of freezes. Essaili et al. present a pro-active resource scheduler for DASH [14]. Based on the clients' buffer levels, the scheduler determines the best streaming rate. This reduces video freezes and is more fair among clients. Zahran et al. present a solution with a comparable goal and effect [15]. Begen et al. adapt the video quality based on the probability of video freezes using a precomputed buffer map [16].

In this paper, we assume the existence of a DANE implementation that can exchange information about channel quality, videos stream, and player state (i.e. play-out buffer level) between mobile base station and client. Instead of only looking at the current state of network and video player, we also predict the evolution of channel quality while determining our buffering strategy. Wang et al. present a channel quality predictor based on machine learning techniques [17]. They target real-time applications but not consider a specific application like we do. Colonnese et al. formulate a closed form solution that models channel quality obtain the probability of video freezes [18], but they don't present measures against freezes.

\section{Modeling Channel Quality Behavior}

Data transmission efficiency in mobile networks depends on the channel quality. We anticipate on this by downloading more video content into the buffer when efficiency is high, and play video from the buffer when efficiency decreases. To determine when to start growing/consuming the video buffer, we have to understand the evolution of channel quality. Accordingly, we follow the next three steps:

A) collect an extensive set of real-word channel quality measurements with a mobile application;

B) fit a Markov chain on the collected traces and determine data transmission efficiency for each state;

C) predict data transmission efficiency for future video segments.

\section{A. Collecting channel quality traces}

In LTE networks, UEs determine the channel quality using a reference signal that is broadcasted by the eNodeB. This process is known as channel estimation. One of the parameters that the UE obtains during channel estimation is the socalled Reference Signal Received Quality (RSRQ). The RSRQ reporting range is defined by the $3 \mathrm{GPP}$ from $-20 \mathrm{~dB}$ (very poor channel quality) to $-3 \mathrm{~dB}$ (excellent channel quality) [19].

To the best of our knowledge, no large and consistent dataset that includes RSRQ parameters exists. Therefore, we developed a smartphone application for the Android platform to measure LTE downlink channel quality. Our app uses the Android 8.0 Telephony API $^{1}$ to gather LTE statistics at

\footnotetext{
${ }^{1}$ https://developer.android.com/reference/android/telephony/package-
} summary.html (accessed March 20, 2018) 
intervals of two seconds. This interval is the lowest update frequency that the platform provides. RSRQ is subject to frequent small changes as a result of interference and multipath fading. Therefore, UEs internally determine RSRQ (and other parameters) every two milliseconds. Nevertheless, the Telephony API gives a good summary of the last two seconds. Small changes would have little to no effect on downloading video segments of a few seconds. The Telephony API reports RSRQ as discrete values within the RSRQ reporting range.

We used two Google/LG Nexus $5 \mathrm{X}^{2}$ smartphones for collecting the traces. We measured LTE channel quality in traces of five minutes in several different environments (e.g. urban, rural), under different speed profiles (e.g. walking, car) and with a varying number of people around us (e.g. village, city). In total, we have collected 377 real-world traces.

\section{B. Modeling channel quality and data rate behavior}

Through analysis of the channel quality traces we obtained how RSRQ evolves over time. Based on this information, we construct a Markov chain to fit this process. The state space $\mathcal{S}$ is defined as eighteen states representing the RSRQ reporting range in steps of one decibel. Transitions in the Markov chain express changes in RSRQ. We observed in our traces that RSRQ can change between any two levels. Therefore, we use a fully connected Markov chain. Nevertheless, small changes in RSRQ are more probable. The transition probability matrix $P$ is obtained through a straightforward mapping between from number of channel quality changes we have observed in the traces to the transition probabilities. We opt for a transition interval of $1 / 3$ second. The two-second intervals from our channel quality traces are too coarse to express the download process of DASH video segments at different buffering speeds. A too small transition interval would cause long execution times when deriving our strategy by solving the MDP that we will describe in the next section.

For each state in the Markov chain, the data transmission efficiency that corresponds with the RSRQ is computed. We follow a process that is similar to how the modulation settings (which determine the efficiency) in LTE networks are established. In the first step, we map RSRQ to Signal to Noise and Interference Ratio (SINR). A theoretical mapping between RSRQ and SINR exists, and is approximated using the following function [19], [20]:

$$
S I N R=\frac{1}{\frac{1}{12-R S R Q}-\rho},
$$

where $\rho$ is the load of the serving cell. We use $\rho=1 / 6$, which indicates a lightly loaded cell. Given the SINR, we use a lookup table to obtain the Channel Quality Indicator (CQI) and corresponding data rate. The data rate is the fraction of bits in a Resource Block (RB) that remains after demodulation (i.e. the actual data). Table I lists the mapping from RSRQ to the data rate. The function $d(x)$ applies this mapping and provides the effective data rate for state $x$.

\footnotetext{
${ }^{2}$ http://www.lg.com/in/lg-nexus-5x/specification.jsp (accessed January 23,
}

Table I

MAPPING RSRQ TO EFFECTIVE DATA RATE

\begin{tabular}{rrrr}
\hline RSRQ (dB) & SINR (dB) & CQI & Eff. data rate \\
\hline-20 & -9.12 & 0 & 0.000 \\
-19 & -8.10 & 0 & 0.000 \\
-18 & -7.07 & 0 & 0.000 \\
-17 & -6.03 & 1 & 0.026 \\
-16 & -4.98 & 1 & 0.026 \\
-15 & -3.92 & 2 & 0.039 \\
-14 & -2.85 & 2 & 0.039 \\
-13 & -1.75 & 3 & 0.063 \\
-12 & -0.06 & 3 & 0.063 \\
-11 & 0.54 & 4 & 0.101 \\
-10 & 1.76 & 4 & 0.101 \\
-9 & 3.05 & 5 & 0.147 \\
-8 & 4.45 & 6 & 0.198 \\
-7 & 6.00 & 6 & 0.198 \\
-6 & 7.82 & 7 & 0.248 \\
-5 & 10.13 & 9 & 0.404 \\
-4 & 13.70 & 10 & 0.459 \\
-3 & INF & 15 & 0.930 \\
\hline
\end{tabular}

\section{Predicting transmission efficiency}

Given the RSRQ Markov chain and the mapping to effective data rate, we can make predictions on how the data rate will evolve. We will use those predictions to estimate how many resources will be required to download the next video segment at a certain speed (i.e. grow, maintain, or shrink the video buffer). Depending on the download speed, downloading a video segment may to take longer than one interval in our model. We compute the multi-step transition probabilities to determine how RSRQ and data rate change while downloading this video segment. Given transition probability matrix $P$, we obtain the $n$-step transition probabilities by multiplying $P$. We denote the probability that our RSRQ model transitions from state $x$ to $y$ in $n$ steps as $P_{x, y}^{n}$.

Driven by the changes in RSRQ that can occur during one segment download, the effective data rate may also vary. To get the expected network resources needed for one video segment we need to estimate the data rate during that download. Therefore, we average the data rates for each possible path between two states, weighing each path by its probability of taking it. We compute the expected data rate when transitioning from state $x$ to $y$ in $n$ steps as follows:

$$
D_{x, y}^{n}=\frac{1}{n} \sum_{i=1}^{n} \sum_{z \in \mathcal{S}} \frac{\left\lceil P_{z, y}^{n-i}\right\rceil P_{x, z}^{i}}{\sum_{w \in \mathcal{S}}\left\lceil P_{w, y}^{n-i}\right\rceil P_{x, w}^{i}} d(z) .
$$

For each of the $n$ steps, we compute the expected data rate in that step, which is the average data rate of all states, weighted by the probabilities that a route from $x$ to $y$ traverses a state in the given step. However, not all paths provide a viable route $x \rightarrow y$, especially when the number of steps becomes small. Therefore, we only consider transitions that after a transition have enough steps left to reach destination state $y$. For each sub-state $z$ in Equation (2), we only include $z$ when the probability of transitioning $z \rightarrow y$ in $n-i$ steps 
is non-zero. Since not all states are included, we adjust the weights accordingly.

\section{Channel Quality Based Buffer Strategy}

The number of network resources that are required for downloading one segment can be estimated with $D_{x, y}^{n}$ and the current channel conditions. How many intervals are needed to download one segment depends on how fast it should be downloaded. Assuming a segment with a duration of two seconds, downloading it in two seconds would maintain the buffer level. Downloading faster increases the buffer, while downloading slower than two seconds (or not at all) would shrink the buffer. We define six different buffering actions (denoted as Bxxx) for our CQBS strategy:

- Growing the buffer: Downloading video segments two (B200) or three (B300) times faster than playing segments out.

- Maintaining the buffer: Download one video segment takes the same time as playing one segment (B100). This action will be chosen over B200/300 when the buffer reaches its maximum level.

- Shrinking the buffer: Relieving the network when bandwidth becomes expensive. We download video segments either at $\frac{1}{3}$ (B033) or $\frac{1}{2}$ (B050) of the playback speed.

- Not downloading: Data transmissions are sometimes not possible because channel quality it too low. During those periods, no video segments will be downloaded and no resources are required $(B 000)$.

Depending on the buffering action, downloading one twosecond segment will take between two and eighteen intervals of $1 / 3$ second. The channel quality of the client and the current buffer fill level determine the best buffer action at each time. We formulate this problem, which is the core of CQBS, as an MDP. Let $\left(\mathcal{S}^{\prime}, A, P^{\prime}, R, \gamma\right)$ be our MDP. The state space $\mathcal{S}^{\prime}$, describes the state of a single video player instance. A state is defined as a couple $(c, b)$ combining the current channel quality (indicated by RSRQ) with the current buffer level. Buffer fill levels are modeled as the number of video units with a duration $1 / 3$ second, aligned with the transitions intervals in our Markov chain. As such, one video segment of two seconds consists of six video units. The buffer is limited to 60 seconds.

The set of actions $A$ covers the six buffering actions, $B 000$ to $B 300$. Based on the buffering action, and the download time of one segment given that action, certain transitions within $\mathcal{S}^{\prime}$ are possible. For example, downloading a two-second segment at speed $B 200$ takes three steps. While downloading six video units, the video player plays out three units. The buffer grows with three units. For buffering action B200, transitions are possible to all states in $\mathcal{S}^{\prime}$, such that $b \rightarrow b+3$. The probabilities for these transitions are obtained from the 3-step transition probability matrix of the RSRQ Markov chain, $P_{x, y}^{3}$. Following this principle, the transition matrix $P^{\prime}$ is build for each combination of RSRQ and buffer level.

The rewards for each transition, $R$, are in general linked to the expected cost in the network. For each action and transition, we compute the expected load on the network as the percentage of resource blocks required by the video player. We calculate the expected load as follows:

$$
l d(x, y, n)=\frac{B \cdot 3 T_{\text {segment }}}{D_{x, y}^{n}} \cdot \frac{1}{\frac{1}{3} R b s \cdot n}
$$

where $B$ is the video bitrate, $T_{\text {segment }}$ the duration of a video segment in seconds, $R b s$ the number of resource blocks that is available per second, and $n$ the number of steps that the segment download takes. Whether the load is used in the reward depends on the following three step process:

1) Check if the buffering action is possible. Only actions that don't cause an overload on the system $(l d(x, y, n)>$ $1)$ are accepted. Also the $B 000$ action is restricted to only being used when channel quality is too low for data transmission. A high negative reward (-10000) to ensure invalid actions.

2) Check if the buffering action would lead to an empty buffer. In case the buffer becomes empty, a penalty of -50 times the duration of the freeze (in ticks) is assigned as a reward for the transitiotns

3) When 1) and 2) don't apply the inverse load (1 $l d(x, y, n))$ is used as reward.

We solve the MDP using the value iteration algorithm with discount factor $\gamma=0.999$ from the Python-based mdptoolbox ${ }^{3}$. The resulting policy is the optimal buffering strategy. For each channel quality and buffer level the strategy provides the buffering action (BOOO to B300) to take. A visualization of our strategy is shown in Figure 1. The lighter the color is in the visualization the faster should be downloaded. The strategy reflects the intuition behind CQBS. When the network resources becomes expensive, the buffering actions that shrink the buffer are selected. Only when the buffer level is low, the buffer has to grow, or has to be maintained as a minimum. As such, the strategy avoids the penalty for an empty buffer. When network traffic becomes cheaper, the strategy lets the buffer grow. The maximum fill level of the buffer grows with the channel quality. This

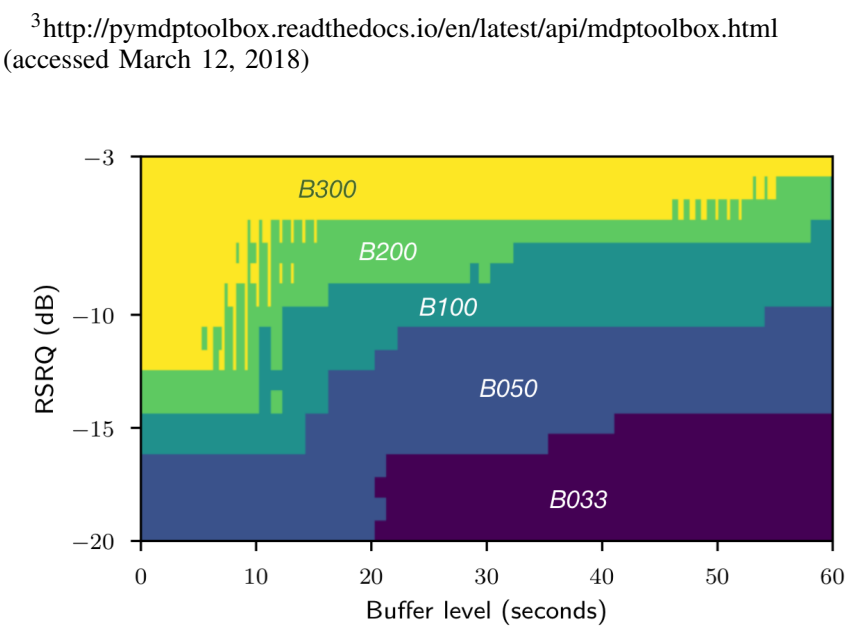

Figure 1. Buffering strategy for constant video quality (1458 Kbit/s), best buffering action for each channel quality and buffer level. 


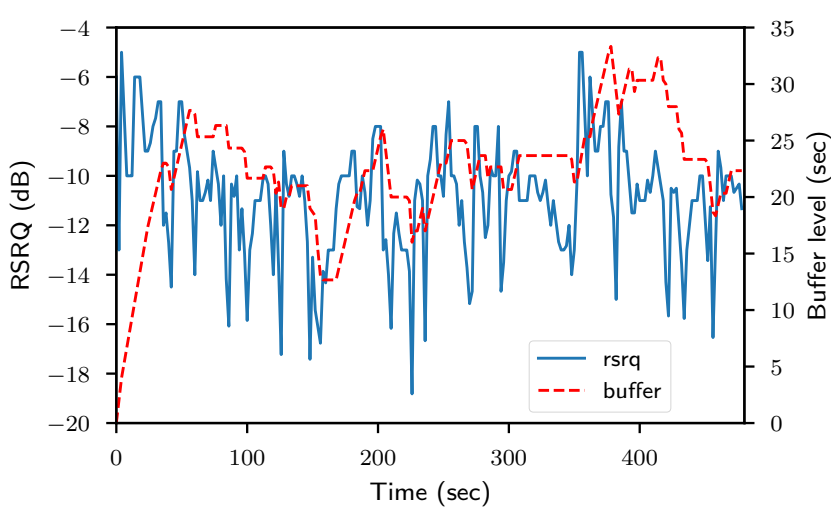

Figure 2. Example operation of CQBS: interaction between channel quality and buffer level.

means that the strategy stops filling the buffer at some point. Only when the channel quality further increases the strategy continues to grow the buffer.

Figure 2 shows an example streaming session with the channel quality and the buffer level overlaid in the same plot. Initially, CQBS grows the buffer when channel quality is good. Around, $t=60$, the signal quality decreases and more video from the buffer is used. The buffer level is restored when signal quality restores. Around $t=350$, the signal quality becomes excellent. This triggers CQBS to fill the buffer to a higher level than it did before.

\section{Performance Evaluation}

In this section, we present the simulation results that compare CQBS to two traditional adaptation algorithms and a naive constant video quality strategy. First, we detail the simulation setup. Then, we compare different algorithms in simulations where channel quality behavior is retrieved from the Markov chain from Section III. The goal of this step is to check the concept behind CQBS, while assuming that CQBS has accurate probabilities on how channel quality behaves. Last, we cross-validate CQBS against our real-word channel quality traces to access the practical performance.

\section{A. Simulation setup}

We look into the performance of CQBS from the perspective of a single client (i.e. how many resources would be required to execute the strategy). We assume that the video streaming client gets the resources assigned that it needs. We use discrete event simulations to simulate an LTE network and a video streaming application. For simplicity, the simulation covers a single video node that is associated to one LTE base station. The LTE base station is configured with $15 \mathrm{MHz}$ bandwidth and has 75,000 RBs available per second. In this section, we denote the network load as the fraction of those RBs that are used by our video streaming application. The channel quality (and changes in channel quality) between the LTE base station and mobile client are either based on the RSRQ Markov chain from Section III or are obtained from our real-world channel quality traces.
The video player that runs on the client node simulates the download of a DASH video stream. A video clip is taken from the movie Sintel $^{4}$ and encoded in ten representations ${ }^{5}$ (i.e. combinations of bitrate and resolution). The video is segmented for DASH with a segment size of two seconds. The video player informs the LTE base station about it its current buffer level and channel quality. The player signals the base station every time before downloading the new video segment. The base station executes our CQBS strategy by combining the buffer level with the current channel quality, selecting the best buffer action and allocating appropriate resources.

As part of this evaluation, we compare four different adaptation and buffering strategies:

1) Conventional: The conventional algorithms uses the download speed of previous video segments to estimate future bandwidth. The video quality will be the highest bitrate that is below this estimation.

2) BOLA: An implementation of the Buffer Occupancybased Lyapunov Algorithm from Spiteri et al. [4]. We use a version that reduces video quality oscillations, comparable to the reference implementation in the DASH.js ${ }^{6}$ player.

3) Static: A naive implementation that produces static video quality. Until the buffer is full, resources for twice the video bitrate are allocated to quickly growing the buffer and prevent freezes. When the buffer is full, resources matching the video bitrate are allocated. When the video bitrate exceeds the networks capacity (e.g. when signal quality is very low), the maximum amount of resources are allocated.

4) CQBS: Adjusts its buffer based on the current channel quality and buffer level. Resources between one thirdand three times the video bitrate are allocated, depending on the selected buffering action. When the video buffer drops below four seconds (i.e. two video segments), and the channel quality does not permit streaming in the target quality, the video player temporarily lowers its video quality, as part of a failover mechanism to ensure uninterrupted playback.

We want to compare the four algorithms for different video quality levels. The conventional algorithm and BOLA always try to get the highest possible video quality, for which they require many network resources. To create a fair comparison between the algorithms - where fair means that the average video quality is the same for each algorithm - we cap the network resources for the conventional algorithm and BOLA as specified in Table II. The resource limits are specified as the maximum fraction of RBs that can be used by a video streaming client.

\footnotetext{
${ }^{4}$ https://durian.blender.org (last accessed: February 20, 2017)

5296Kbit/s@240p, 395Kbit/s@240p, 493Kbit/s@360p, 732Kbit/s@360p, 971Kbit/s@480p, 1.458Kbit/s@480p, 1.934Kbit/s@720p, 2.878Kbit/s@720p 3.779Kbit/s@1080p, 5.544Kbit/s@1080p

${ }^{6}$ https://github.com/Dash-Industry-Forum/dash.js/wiki (accessed: March 5, 2018)
} 
Table II

NETWORK RESOURCE LIMITS AS A FRACTION OF THE TOTAL AVAILABLE RBS

\begin{tabular}{lrrrrrrrr}
\hline Target video quality level & $\mathbf{3}$ & $\mathbf{4}$ & $\mathbf{5}$ & $\mathbf{6}$ & $\mathbf{7}$ & $\mathbf{8}$ & $\mathbf{9}$ & $\mathbf{1 0}$ \\
\hline Conventional adaptation limit & 0.063 & 0.095 & 0.140 & 0.200 & 0.280 & 0.400 & 0.650 & 1.00 \\
BOLA limit & 0.045 & 0.072 & 0.110 & 0.156 & 0.216 & 0.305 & 0.435 & 1.00 \\
\hline
\end{tabular}

\section{B. Performance comparison based on the channel quality} behavior model

In the first part of the evaluation we simulate channel quality behavior based on random walks in the RSRQ Markov chain from Section III. The CQBS strategy is based on the same Markov model, and thus it is based on accurate probabilities how RSRQ can change. As such, CQBS will perform optimal, allowing us to check the concept behind our strategy. Per setting, we generate 25,000 channel quality traces. For each trace, we simulate one 10-minute DASH streaming session, which we will call an instance. For each instance, we measure the video quality (levels ranging from 1 to 10) and load on the network. A video quality level is used instead of the video bitrate. The encoding settings of our video are chosen such that every step gives a comparable increase in quality. However, the bitrate that is needed for each step increases with the video quality. Averaging over video bitrate would give skewed results.

First, we looked into the differences in video quality between the four algorithms. The target video quality is level five. For each instance we computed the mean video quality. The distribution of mean video qualities is shown in Figure 3. The boxes in Figure 3 indicate the four quartiles. The red line in the box in the median. For the static and CQBS strategies, Figure 3 reveals that the overall video quality matches the target quality in almost all instances. The exceptional instances suffered from either low channel quality at the startup or long periods of low channel quality. As result, the fallback mechanism temporarily lowered the video quality. For the conventional and BOLA algorithms the mean video quality lies within one and a half video quality levels around the target.

The video quality within one instance is also not constant

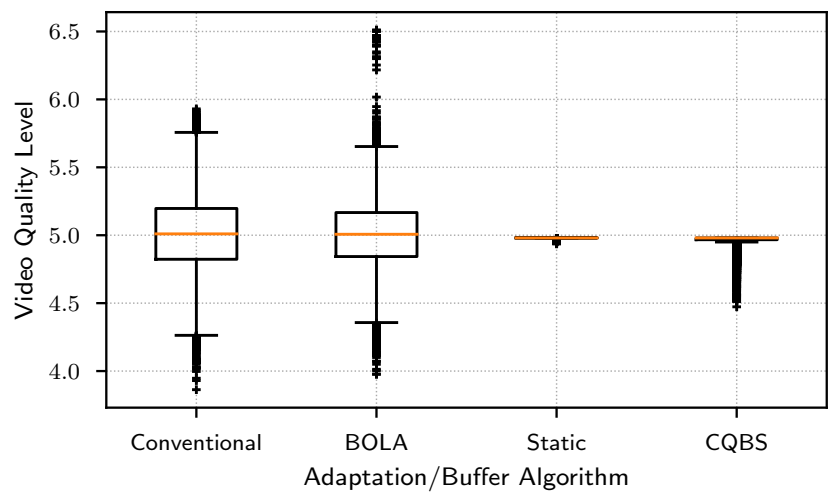

Figure 3. Distribution of mean video quality per instance, target video quality level five. for the conventional and BOLA algorithms. These adaptation algorithms adapt the video quality to match the network conditions. Because the network conditions change (as a result of the channel condition chaning), the video quality changes as well. The cumulative distribution of video quality within instances is shown in Figure 4. The figure shows that the video qualities are spread out over all levels, as can be seen by comparing the dashed lines to the solid lines in Figure 4 .

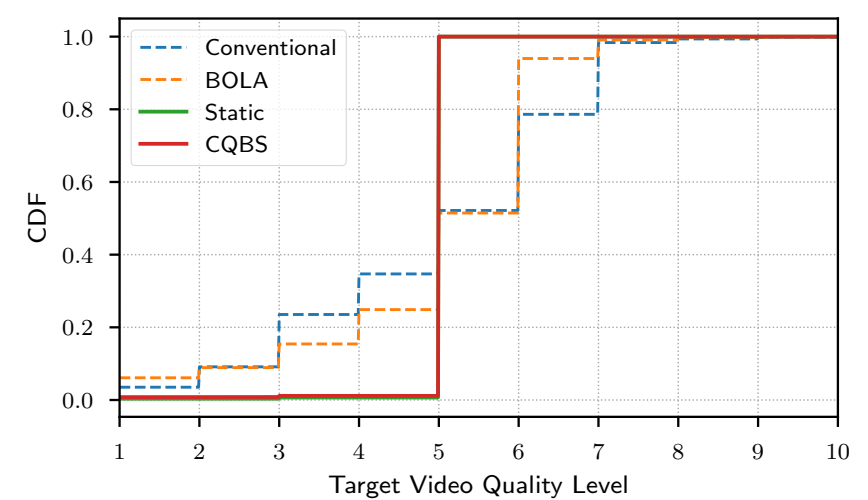

Figure 4. Cumulative distribution of video quality within the instances, target video quality level five.

When using the conventional and BOLA algorithm, the video is streamed in the target quality only a relatively small fraction of the time. The broad spread of video qualities during each run indicates that the video quality changes from time to time. In Figure 5, we observe that both the conventional algorithm and BOLA show many video quality switches. The conventional algorithm performs worst, resulting in more than 12 quality switches per minute for most of the target quality levels. BOLA perform better, but the switching frequency is still high. A big difference can be observed from target quality level 10. In this case, all algorithms had the full spectrum available for streaming. In the case of BOLA, it meant that it could maintain large buffers. Due to the nature of BOLA, an almost full buffer will result in video segments of the highest quality. Even when the signal quality decreased, the buffer levels were still sufficient for BOLA to request video quality level 10. As the result, BOLA worked similar (except during the startup phase) to the naive static strategy. This resulted in little quality switches, but to an increased network load as we will discuss next in this section.

The high number of quality switches when using the traditional algorithms may be perceived by the user as annoying. In comparison, the naive static quality strategy and CQBS maintain an almost perfectly constant video quality. 


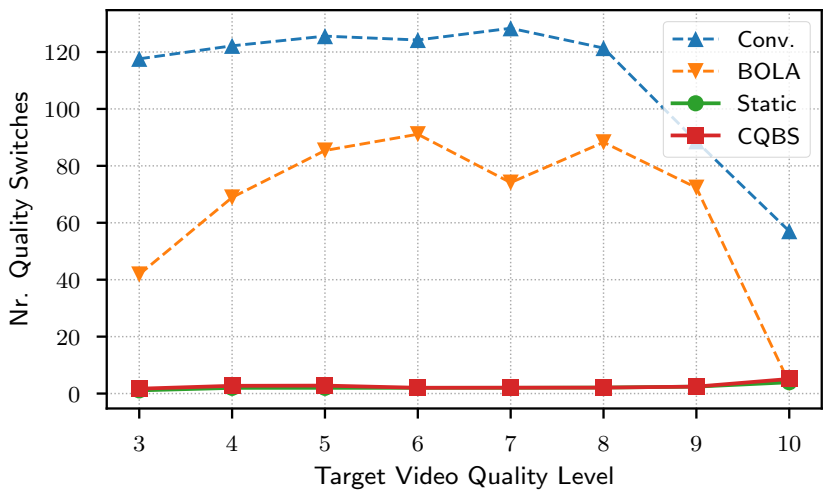

Figure 5. Number of quality switches as a function of video quality.

Although providing a constant video quality may be more beneficial for the end-user, it could increase cost for the network operator. Figure 6 shows the distribution of network load (percentage of RBs that were used for video streaming) per instance. For most of the instances, the network load for the conventional algorithm and BOLA is comparable. Nevertheless, BOLA shows some instances that have relatively high load. Comparing the medians of the traditional algorithms with the naive static quality strategy, we observe and increase in network load of $25 \%$. This increase in network load takes up resources that cannot be used by other clients in the same cell. The increase makes a difference over ten minutes, from up to $2 \mathrm{Mbit} / \mathrm{s}$ bandwidth for clients with good signal quality, to about $330 \mathrm{Kbit} / \mathrm{s}$ for an average client. This bandwidth cannot be used anymore for several Web browsers, a high quality music stream, or a low quality video stream.

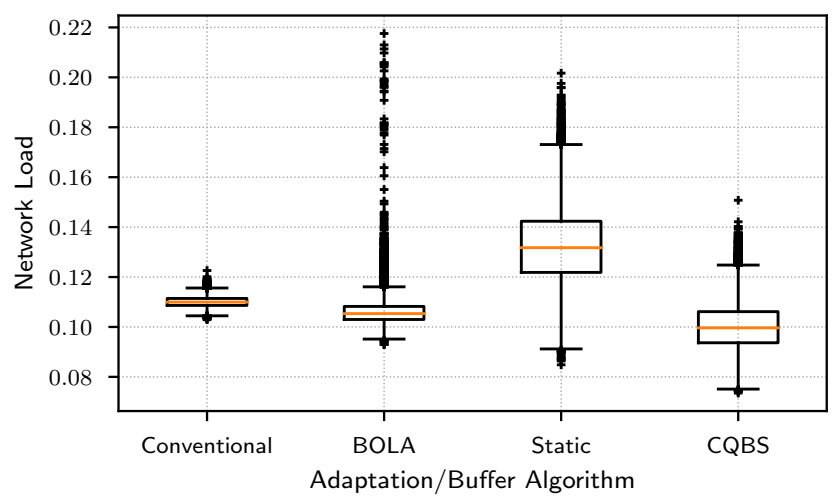

Figure 6. Distribution of overall network load per instance, target quality level 5.

Our CQBS strategy is designed to reduce the load on the network. It consumes video from the buffer when network bandwidth is expensive. When channel quality becomes better and bandwidth comparatively cheap, it re-fills the video buffer. Figure 6 shows the impact of this strategy on the network load. On average, the use of network resources by CQBS is on the same level as the traditional adaptation algorithms. In $23 \%$ to
$64 \%$ of the cases, CQBS requires less resources than the most efficient instance from the conventional algorithm and BOLA.

Figure 7 shows the difference in network usage per video quality level for the four different implementations. The differences are shown relative to the network resources used by CQBS. CQBS significantly reduces the network load compared to non-optimized static quality implementation. On average, the network load is $19 \%$ lower when using CQBS. When comparing CQBS to the conventional algorithm and BOLA, we see overall a similar network load for the three strategies.

For quality level 9 , the conventional algorithm yields a high network load. The conventional algorithm bases the quality of future video segments on the network throughput in the near past. Past performance is thus not a good indicator for the future. In this case, the conventional algorithm overestimated the quality and had to recover the buffer afterwards. The reason for the higher load for BOLA at quality level 10, is given earlier this section.

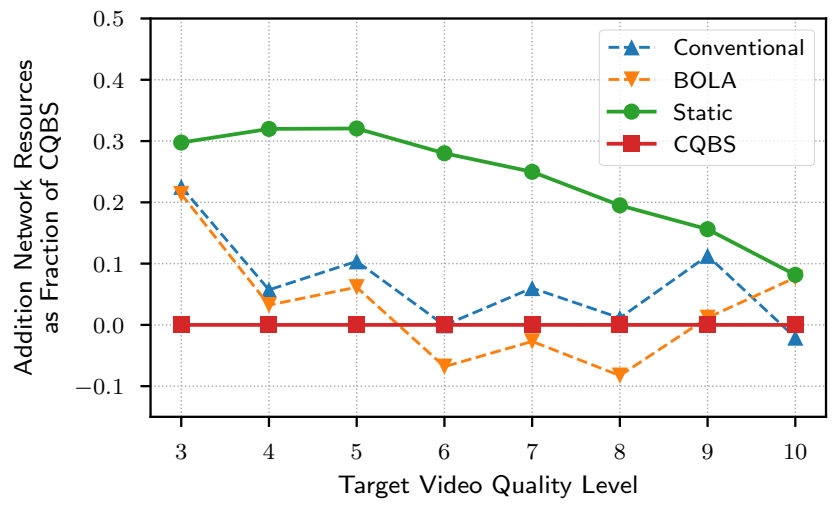

Figure 7. Additional usage of network resources relative to the resources used by CQBS.

\section{Validation against real-world traces}

In the second part of the evaluation, we validate our CQBS algorithm against the real-world LTE traces that we have collected. We use the cross-validation technique, where our strategy was trained on $80 \%$ of the traces and then tested against the remaining $20 \%$ of the LTE traces, where the fraction of traces that is used for validation rotates. This sums up to 377 instances per setting. The DASH player streams a 8:20 minutes clip from the movie Sintel using the same encoding settings as before. The LTE channel quality traces have a duration of five minutes. Therefore, we repeat a trace to cover the full length of the video.

In general, the performance of CQBS and the other algorithms is similar when using the real-world traces instead of the model-based traces. Therefore, we will focus on the differences that we have observed, starting with the stability of the streams' qualities. Figure 8 shows the distribution of mean video quality for each instance. Given target quality level 7, the constant video strategies perform well, having almost 


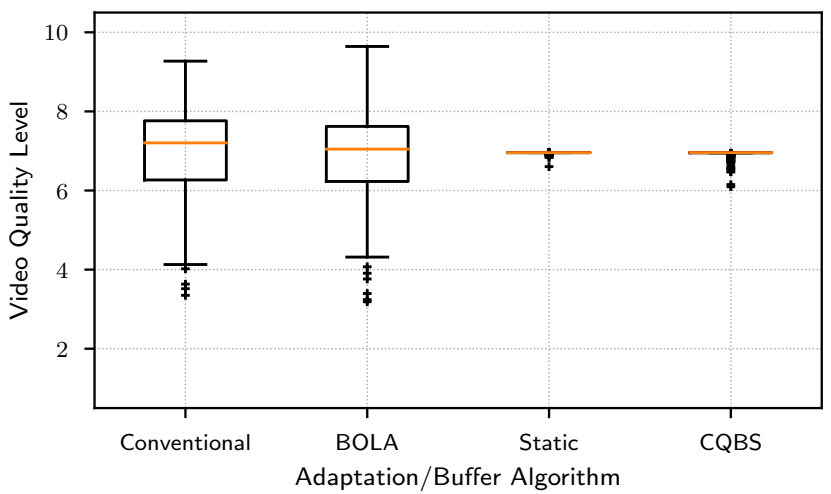

Figure 8. Distribution of mean video quality per instance, target video quality level seven.

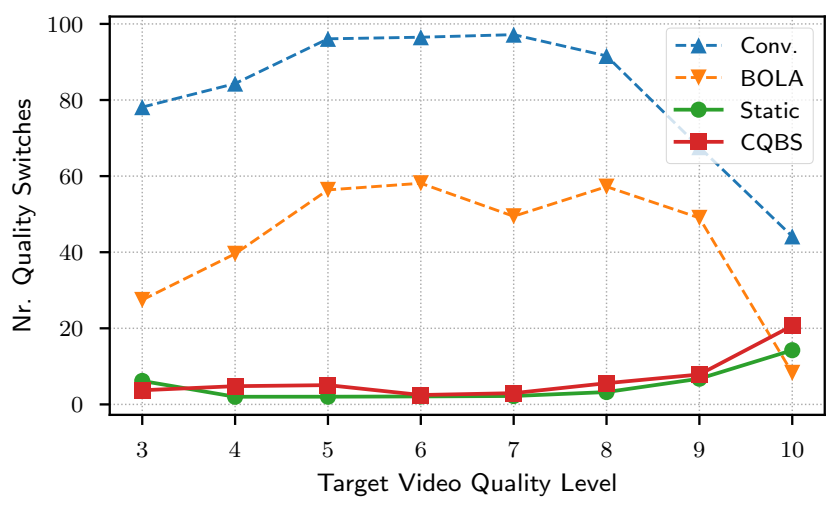

Figure 9. Mean number of quality switches as a function of video quality.

all instances at the target video quality. For the conventional algorithm and BOLA the spread of average video quality is larger. With video resolutions ranging between SD (360p) and Full-HD (1080p) the differences between instances are large, indicating that reserving a fixed portion of network resources cannot provide guarantees on the video quality.

With regards to the number of quality switches, we don't observe a difference for the conventional algorithm and BOLA (the video was slightly shorter, but the number of switches is consistently lower). In contrast, our CQBS strategy produces a higher switching frequency, especially for the high target qualities. In CQBS, quality switches only occur when channel quality is bad and the buffer level is too low to guarantee uninterrupted streaming. This indicates that buffers levels get lower more often because the duration and severity of low channel quality is underestimated by our model. Our model is thus not entirely accurate at low channel qualities. However, even though there is a slight increase in quality switches, CQBS still greatly outperforms the traditional algorithms.

In Figure 10, the differences in network resource usage relative to CQBS for the target quality levels are shown. Compared to the model-based traces, the network loads for the real-world LTE traces show small differences. There is a slightly smaller difference in network load between the naive static strategy and CQBS. The naive static strategy seems to perform slightly more efficient given the real-world traces. Nevertheless, our CQBS strategy is able to decrease

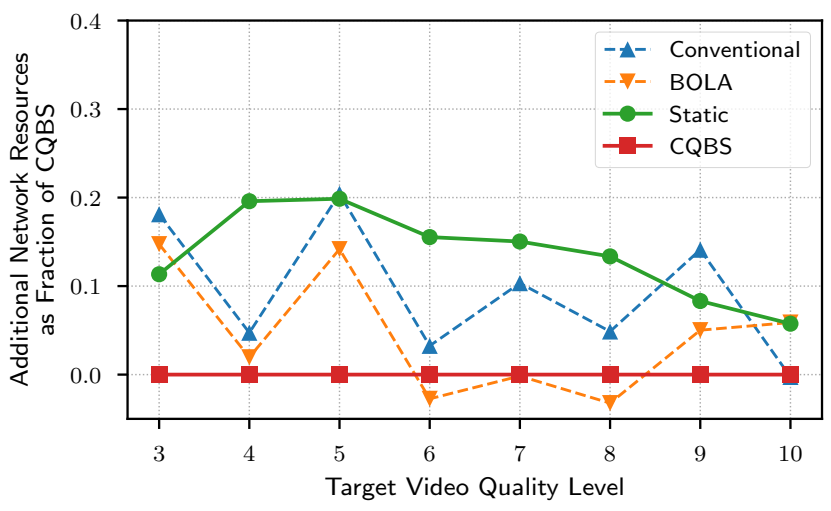

Figure 10. Additional usage of network resources relative to the resources used by CQBS.

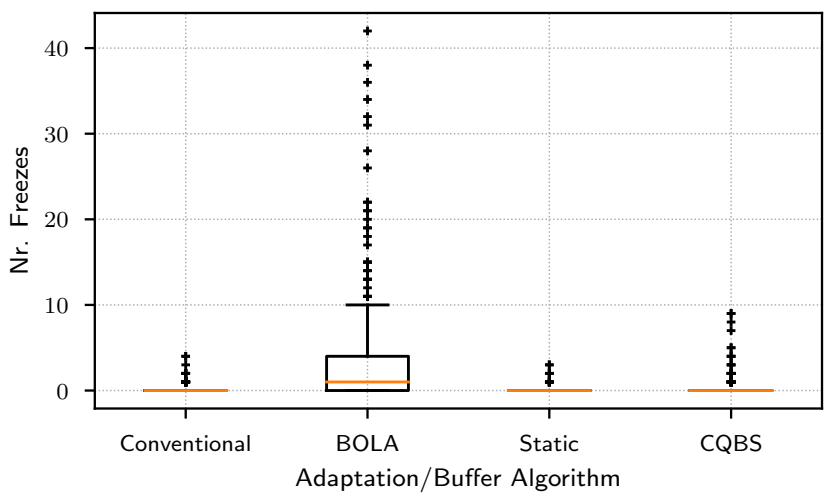

Figure 11. Distribution of the number of video freezes per instance, target quality level 7.

the network load by on average $15 \%$. In terms of network load, CQBS performs slightly better than the conventional algorithm, and on average as good as BOLA.

Although BOLA performs overall best when it comes to network load, we did observe a high number of freezes when using BOLA. In Figure 11, it can be seen that BOLA has a structural problem and that freezes occur in most of the instances. In a large number of runs, the number of freezes can even be considered as very high. A high number of freezes is disastrous for the user experience, and will lead to abandonment of the video stream [6]. The other algorithms, including CQBS, do no have playback interruptions in almost all the runs. Only in exceptional cases freezes might occur. This shows that even though CQBS actively reduces the buffer level, it does not compromise the continuity of the stream.

Overall, we can conclude that CQBS can maintain a constant video quality. It outperforms the traditional adaptation algorithms that show fluctuations in video quality as a result of variations in LTE channel quality. Compared to high number of freezes in BOLA, CQBS is able to stream without interruptions in almost all runs. As such, CQBS provides the best experience to the user. When looking at network load, CQBS performs on par with the traditional algorithms, but outperforms the non-optimized static quality strategy. 


\section{CONCLUSION \& FUture WORK}

Online video streaming is one of the biggest contributors to data consumption on mobile networks. Because of the high data volumes and the mobile networks' high throughput variations, it is a difficult practice to deliver high quality video to the end-user. Video streaming players adapt the video quality to the networks' conditions. However, the common variations in LTE channel quality can cause large fluctuations in video quality during playback, which may be perceived by users as annoying. The CQBS buffering strategy that we present in this paper allows to deliver near-constant video quality to mobile clients without an increase in costs, compared to the traditional adaptation algorithms. The method that we use to derive the optimal buffering strategy is elegant. At its base, the state space of the Markov chain that drives our MDP consists of only two dimensions: RSRQ channel quality and buffer level. The results show that this relatively simple model already yields good performance, and thus models the environment well. Furthermore, channel quality and buffer level can both be measured by the client device, which is important when implementing CQBS in practice.

In a practical deployment, channel quality, video quality and buffer level should be send to the base station. Exchanging CQIs is already part of the LTE protocol and will continue to exists in future $5 \mathrm{G}$ networks. Exchanging information about video quality and buffer level is not default in DASH-based streaming. To realize our strategy we rely on DASH Aware Network Elements. These network elements coordinate DASH players and manages network resources. In our case, the DANE will be located in the mobile base station where it executes our CQBS strategy. Communication between DANE and DASH player will follow the standardized interactions as described in Server and Network Assisted DASH [9], [21]. Given the standardization efforts we expects DANEs to become more common, especially in future $5 \mathrm{G}$ deployments.

In future work, we will look into scenarios with multiple video players in a mobile cell. We will investigate the interactions between concurrent clients. We are interested in the impact of concurrent clients on the video streaming applications and the implications for the mobile operator (i.e. how can the mobile operator better divide the available networks resources among the video clients). In addition, we will investigate further improvement of CQBS by extending the state space of the Markov chain. We have already explored the effect of including trends in channel quality into our model, in particular upward versus downward trends. Our first results show slightly better performance than CQBS, but less than expected. It appears that in most of the cases, CQBS selects the same buffering action as this initial model enhancement. Differences mostly occur at the edges of the state space, which, however, are rarely reached.

\section{REFERENCES}

[1] Sandvine, Inc, "Global internet phenomena report," Tech. Rep., 2016.

[2] "ISO/IEC 23009-1 Information technology - Dynamic adaptive streaming over HTTP (DASH) - Part 1: Media presentation description and segment formats," Tech. Rep., 2014.
[3] S. Akhshabi, A. C. Begen, and C. Dovrolis, "An Experimental Evaluation of Rate-adaptation Algorithms in Adaptive Streaming over HTTP," in Proceedings of the Second Annual ACM Conference on Multimedia Systems. New York, NY, USA: ACM, 2011, pp. 157-168.

[4] K. Spiteri, R. Urgaonkar, and R. K. Sitaraman, "BOLA: Nearoptimal bitrate adaptation for online videos," in IEEE INFOCOM 2016 - The 35th Annual IEEE International Conference on Computer Communications, Apr. 2016, pp. 1-9.

[5] T. Hoßfeld, M. Seufert, C. Sieber, T. Zinner, and P. Tran-Gia, "Identifying QoE optimal adaptation of HTTP adaptive streaming based on subjective studies," Computer Networks, vol. 81, pp. 320-332, 2015

[6] F. Dobrian, A. Awan, D. Joseph, A. Ganjam, J. Zhan, V. Sekar, I. Stoica, and H. Zhang, "Understanding the Impact of Video Quality on User Engagement," Commun. ACM, vol. 56, no. 3, pp. 91-99, Mar. 2013.

[7] S. Lederer. (2015, Feb.) Why YouTube \& Netflix use MPEG-DASH in HTML5. [Online]. Available: https://bitmovin.com/status-mpeg-dashtoday-youtube-netflix-use-html5-beyond/

[8] T.-Y. Huang, N. Handigol, B. Heller, N. McKeown, and R. Johari, "Confused, timid, and unstable: picking a video streaming rate is hard," in IMC '12: Proceedings of the 2012 ACM conference on Internet measurement conference. New York, New York, USA: ACM Request Permissions, Nov. 2012, pp. 225-238.

[9] E. Thomas, M. O. van Deventer, T. Stockhammer, A. C. Begen, and J. Famaey, "Enhancing MPEG DASH performance via server and network assistance," in The Best of IET and IBC. Institution of Engineering and Technology, 2015, pp. 48-53.

[10] N. Bouten, J. Famaey, S. Latré, R. Huysegems, B. D. Vleeschauwer, W. V. Leekwijck, and F. D. Turck, "QoE optimization through innetwork quality adaptation for HTTP Adaptive Streaming," in 2012 8th international conference on network and service management (cnsm) and 2012 workshop on systems virtualiztion management (svm), Oct. 2012, pp. 336-342.

[11] G. Cofano, L. De Cicco, T. Zinner, A. Nguyen-Ngoc, P. Tran-Gia, and S. Mascolo, "Design and Experimental Evaluation of Network-assisted Strategies for HTTP Adaptive Streaming," in Proceedings of the 7th International Conference on Multimedia Systems. New York, NY, USA: ACM, 2016, pp. 3:1-3:12.

[12] J. W. Kleinrouweler, S. Cabrero, and P. Cesar, "Delivering Stable Highquality Video: An SDN Architecture with DASH Assisting Network Elements," in Proceedings of the 7th International Conference on Multimedia Systems. New York, NY, USA: ACM, 2016, pp. 4:1-4:10.

[13] T. Wirth, Y. Sanchez, B. Holfeld, and T. Schierl, "Advanced Downlink LTE Radio Resource Management for HTTP-streaming," in Proceedings of the 20th ACM International Conference on Multimedia. New York, NY, USA: ACM, 2012, pp. 1037-1040.

[14] A. E. Essaili, D. Schroeder, E. Steinbach, D. Staehle, and M. Shehada, "QoE-Based Traffic and Resource Management for Adaptive HTTP Video Delivery in LTE," IEEE Transactions on Circuits and Systems for Video Technology, vol. 25, no. 6, pp. 988-1001, Jun. 2015.

[15] A. H. Zahran, J. J. Quinlan, K. K. Ramakrishnan, and C. J. Sreenan, "SAP: Stall-Aware Pacing for Improved DASH Video Experience in Cellular Networks," in Proceedings of the 8th ACM on Multimedia Systems Conference. New York, NY, USA: ACM, 2017, pp. 13-26.

[16] A. Beben, P. Wiśniewski, J. M. Batalla, and P. Krawiec, "Abma+: Lightweight and efficient algorithm for http adaptive streaming," in Proceedings of the 7th International Conference on Multimedia Systems, ser. MMSys '16. New York, NY, USA: ACM, 2016, pp. 2:1-2:11.

[17] X. Wang, E. Grinshpun, D. Faucher, and S. Sharma, "On Medium and Long Term Channel Conditions Prediction for Mobile Devices," in 2017 IEEE Wireless Communications and Networking Conference (WCNC), Mar. 2017, pp. 1-6.

[18] S. Colonnese, S. Russo, F. Cuomo, T. Melodia, and I. Rubin, "Timely Delivery Versus Bandwidth Allocation for DASH-Based Video Streaming Over LTE," IEEE Communications Letters, vol. 20, no. 3, pp. 586-589, Mar. 2016

[19] H. Holma, A. Toskala, and J. Reunanen, LTE Small Cell Optimization: 3GPP Evolution to Release 13. John Wiley \& Sons, 2016.

[20] C. Ide, R. Falkenberg, D. Kaulbars, and C. Wietfeld, "Empirical Analysis of the Impact of LTE Downlink Channel Indicators on the Uplink Connectivity," in 2016 IEEE 83rd Vehicular Technology Conference (VTC Spring), May 2016, pp. 1-5.

[21] "ISO/IEC 23009-5: Information technology - Dynamic adaptive streaming over HTTP (DASH) - Part 5: Server and network assisted DASH (SAND)," Tech. Rep., 2017. 